\title{
Characterization of novel graphene-like materials prepared by new cheap and environmentally friendly synthetic methods ${ }^{+}$
}

\author{
Dana Němečková ${ }^{1}$, Richard Ševčík ${ }^{1, *}$ and Pavel Pazdera ${ }^{1}$ \\ 1 Department of Chemistry, Masaryk university, Kotlářská 2, Brno, 611 37, Czech Republic \\ * Correspondence: ja_richard@email.cz; Tel.: +420-54949-2677 \\ + Presented at the title, place, and date.
}

Received: date; Accepted: date; Published: date

\begin{abstract}
We report characterization data of novel graphene-like materials prepared by new cheap and environmentally friendly synthetic methods. FT-IR, Raman and X-ray photoelectron spectroscopy, scanning electron microscopy and thermal and elemental analysis methods were used to describe features of novel graphene-like materials and obtained data were compared with the data of commercial standards.

It was found that employment of gentle oxidizing agents supported by ultrasound action instead of harsh oxidizers and strongly acidic solutions provides a-few-layer graphene oxides with low-defect layers in high yields. Although keeping the same level of oxidation like commercial standards new products are more stable due to a lower damage of carbon layers. Obtained products can then be further modified to reduced graphene oxides or amine-modified derivatives. All of the characterization data are presented and discussed in the article.
\end{abstract}

Keywords: graphene; graphene oxide; synthesis; ultrasound; manufacturing; characterization; green chemistry; sustainable chemistry, spectroscopy

\section{Introduction}

Graphene and graphene-like materials passed through a period of a huge interest and intense research in recent years as evidenced by a huge number of publications [1-4]. Although time of boom and often of a non-critical adoration of graphene is behind thus research can now be focused only on the most promising areas of application such as electrical engineering and electronics, energy storage, adsorption and catalysis, medicinal and biochemical applications and material science $[1,3,5,6]$. As the industry standing behind the fields of interest are capable of enormous consumption of graphene-like materials there is still a big effort in research of easy and cheap methods of their synthesis and especially manufacturing. Moreover, particular application often requires a specific features and quality of a graphene-like material which makes the task more challenging.

In this paper we report a detailed characterization data of a-few-layer graphene oxides (GO) which were prepared by a new, cheap and environmentally friendly method from graphite. Both of the synthetic ways follow the principles of green and sustainable chemistry and employ mild oxidizing agents in water supported by ultrasound action. Simple reaction scheme can be easily expanded into industrial scale to yield large batches of graphene oxide. The key aspects of utilization of mild and non-hazardous oxidizing agents can be seen in fact that no toxic waste is formed, and products are obtained in a good quality as their carbon structure is not stressed by harsh oxidizers and highly acidic solutions. Moreover, savings in costs and time can be appreciated especially by potential commercial manufacturers. 


\section{Materials and Methods}

Graphene oxides were prepared by oxidation of graphite using mild oxidizing agents. First, graphite was dispersed in water using ultrasonic bath then a solution of the oxidizer was added dropwise at $60^{\circ} \mathrm{C}$ for 16 hours. Then graphene oxide was filtered off and washed with water to neutral reaction. Subsequently crude product was washed with organic solvents to remove side products and especially low molecular weight carbon fragments. Finally, it was dried to a constant weight at $140{ }^{\circ} \mathrm{C}$.

Process details are a subject of a classified know-how of Masaryk University as an originator.

Reduced graphene oxides were then obtained from graphene oxides using ascorbic acid or hydrazine hydrate as reducing agents at higher temperatures following the known procedures [3,7].

Carbon-oxide functional groups of graphene oxides can be alternatively modified using standard organic chemistry methods, e.g. amination or esterification [3,8].

FT-IR spectra were measured on Bruker Alpha FTIR Platinum-ATR. Raman spectroscopy (micro-spectrometer Horiba LabRAM HR Evolution), scanning electron microscopy (Tescan MIRA3 equipped with a Schottky Field Emission electron gun and with SE (Secondary electrons) and BSE (Back-scattered electrons) detectors), X-ray photoelectron spectroscopy (ThermoScientific ESCALAB 250Xi) and X-ray diffraction analysis (diffractometer Rigaku SmartLab Type F) were performed at R\&D Centre for Low-Cost Plasma and Nanotechnology Surface Modifications (CEPLANT), Department of Physical Electronics, Faculty of Science, Masaryk University, Brno. Thermal analysis was measured at Institute of Technology of Building Materials and Components, Faculty of Civil Engineering, Brno using instrument Mettler Toledo - TGA/SDTA 851e. Elemental analysis was measured at Regional Centre of Advanced Technologies and Materials, Faculty of Science, Palacký University, Olomouc using ThermoScientific Flash 2000 Analyzer.

\section{Results and Discussion}

A series of starting materials (graphite of the flake, expandable or expanded type) and oxidizing agents were tested and investigated. Finally, graphene oxides of a desired quality and yield were prepared using two oxidizing agents which met all of the requirements such as low price, common availability, safe and easy handling and last but not least a low side impact to the structure of carbon plates.

Here we report characterization data of two graphene oxides further denoted as GO I and GO II which have similar features although some differences can be observed as well. For ilustration we compare our products with similar compounds of a commercial supplier Sigma-Aldrich (graphene oxide, cat. no. 796034; denoted as GO-SA and reduced graphene oxide, cat. No 805424; denoted as rGO-SA).

When discussing graphene oxide we should be interested in a level of oxidation. Oxygen content was determined by X-ray photoelectron spectroscopy (XPS) and elemental analysis (EA). Although XPS brings information about oxygen content only from the thin layer of surface, it provides data on specific oxygen containing groups which are present on graphene oxide particle surface. Summarized data are presented in Table 1. It can be seen that all of the graphene oxides have a similar content of oxygen functional groups. GO I and GO II have similar oxygen content as determined by XPS but they differ in distribution of carbon-oxygen groups as GO II is richer in carboxyl groups. Some difference can be additionally observed in a volume distribution of oxygen as elemental formula is quite different in case of GO I indicating more homogeneous distribution of oxygen in the mass of graphene oxide particles. 
Table 1. Graphene oxides - elemental analysis and XPS analysis results.

\begin{tabular}{cccccccc}
\hline & $\begin{array}{c}\text { Formula } \\
\text { (EA) }\end{array}$ & \multicolumn{5}{c}{ C1s, specific groups abundance (rel. \%) } \\
(XPS) \\
\hline & & $\mathrm{C}=\mathrm{C}$ & $\mathrm{C}-\mathrm{C}$ & $\mathrm{C}-\mathrm{O}$ & $\mathrm{C}=\mathrm{O}$ & $\mathrm{C}(\mathrm{O}) \mathrm{O}$ & sum $\mathrm{C} \sim \mathrm{O}$ \\
\hline GO I & $\mathrm{C}_{8} \mathrm{O}$ & 61.96 & 23.66 & 6.69 & 2.62 & 2.69 & $\mathbf{1 2 . 0 0}$ \\
\hline GO II & $\mathrm{C}_{16} \mathrm{O}$ & 63.72 & 24.10 & 5.38 & 1.76 & 4.71 & $\mathbf{1 1 . 8 5}$ \\
\hline GO-SA & $\mathrm{C}_{14} \mathrm{O}$ & 69.88 & 18.52 & 8.69 & 1.24 & 1.67 & $\mathbf{1 1 . 6 0}$ \\
\hline
\end{tabular}

Concerning literature methods $[9,10]$ of graphite oxidation, products mostly suffer from low quality of carbon layers as they are strongly damaged by harsh oxidizing agents such as potassium manganate or dichromate or sodium nitrite in strongly acidic solutions which are employed. In addition products can be contaminated by impurities in a form of low molecular weight carbon fragments which are formed as by-products during oxidation. They are very often intercalated among carbon layers.

Damage of carbon layers is mostly determined using Raman spectroscopy because intesity of the peak at ca $1350 \mathrm{~cm}^{-1}$ (D- peak) is related to disorder in sp $\mathrm{sp}^{2}$ hybridization of carbon atoms in the layers [11,12]. Regular structure of sp2 hybridized carbons gives rise to the G-peak at ca $1580 \mathrm{~cm}^{-1}$. Figure 1 represents Raman spectra of graphene oxides and it is evident that in case of GO I and GO II D-peak intensity is very low compared to that of GO-SA. Thus we can claim that employment of mild oxidizing agents does not cause a significant damage to carbon layers despite the same level of oxidation. Preservation of undamaged regular carbon layers can be considered as a very important aspect because unique features of graphene(-like) materials arise from regular carbon structure and its electronic arrangement. Position and intensity of 2D-peak at ca $2700 \mathrm{~cm}^{-1}$ is then related to the number of layers of a graphene-like materials [11,12]. Spectra show features of a-few-layer graphene oxides produced in a basic arrangement but when it is required, for example due to a further application, we are able to reduce the number of layers using some additives as it is clearly seen in inset of Fig. $1 \mathrm{~b}$.

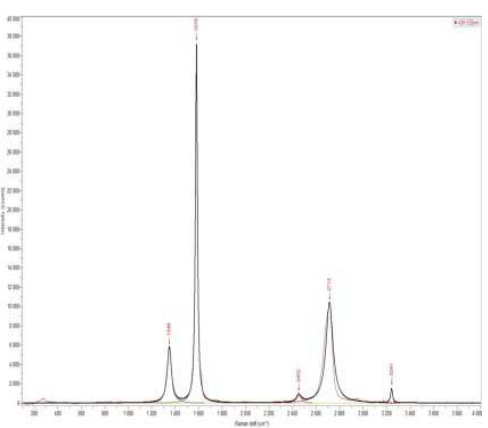

(a)

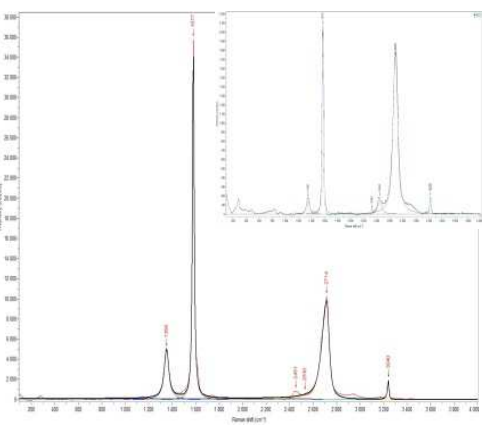

(b)

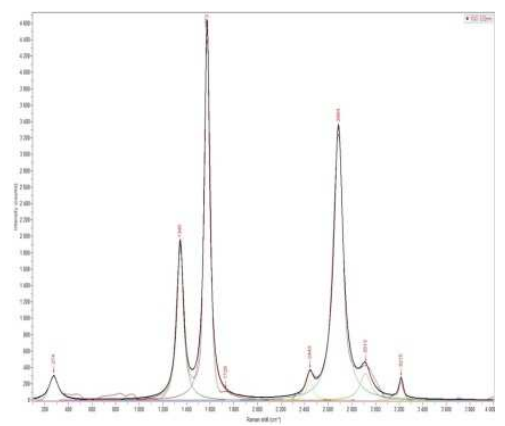

(c)

Figure 1. Raman spectra of graphene oxides: (a) GO I; (b) GO II, inset - product with reduced number of layers and (c) GO-SA.

On the contrary FT-IR spectra of all of the compounds (Figure 2) bring us only brief infromation as they suffer a lot from low intensities. We can hardly observe broad bands belonging to $\mathrm{C}=\mathrm{C}$ bonds (below ca $1650 \mathrm{~cm}^{-1}$ ) and carbonyl/carboxyl groups (above ca $1650 \mathrm{~cm}^{-1}$ ). Not even other measuring techniques were succesful to provide FT-IR spectra of a better quality. 


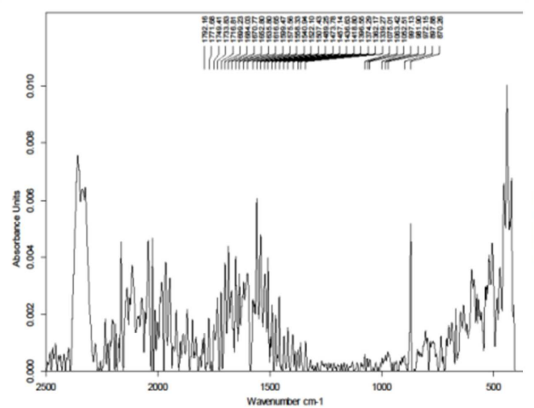

(a)

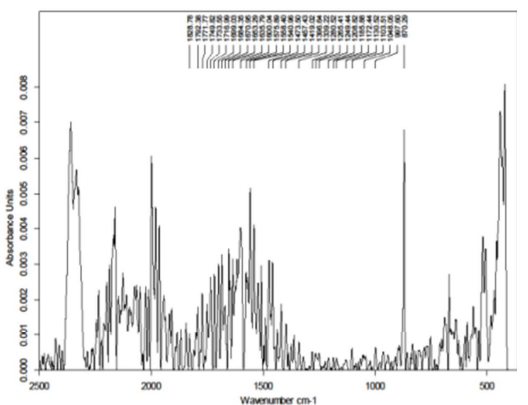

(b)

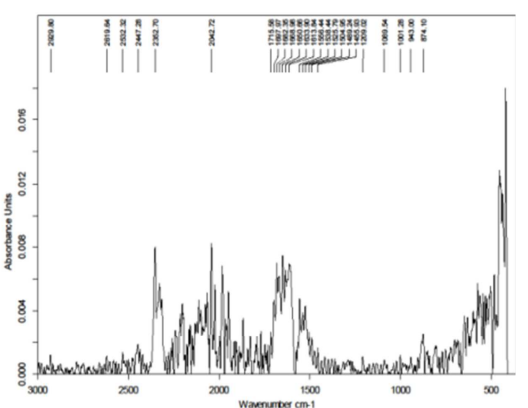

(c)

Figure 2. FT-IR spectra of graphene oxides: (a) GO I; (b) GO II and (c) GO-SA.

Products were also examined using scanning electron microscopy (SEM). When observing SEM images of GO I and GO II (Figure 3) we should highlight the presence of thin and large layers which are not damaged by oxidation. Additionally no low molecular weight fragments are observed on the surface of carbon plates as it can be seen in case of GO-SA product. Larger and undamaged plates of graphene oxides GO I and GO II can be easily ground using ball or vibratory mill if necessary for a further application.

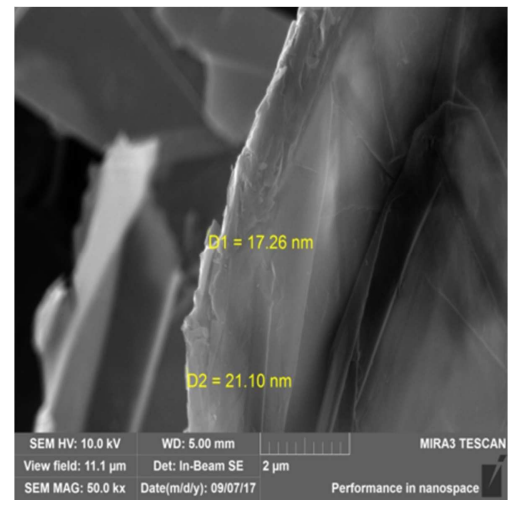

(a)

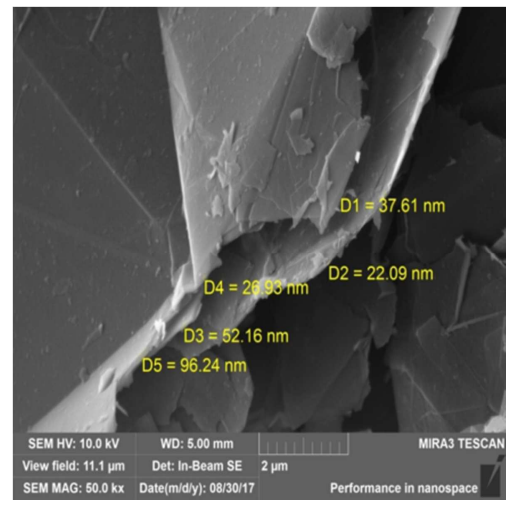

(b)

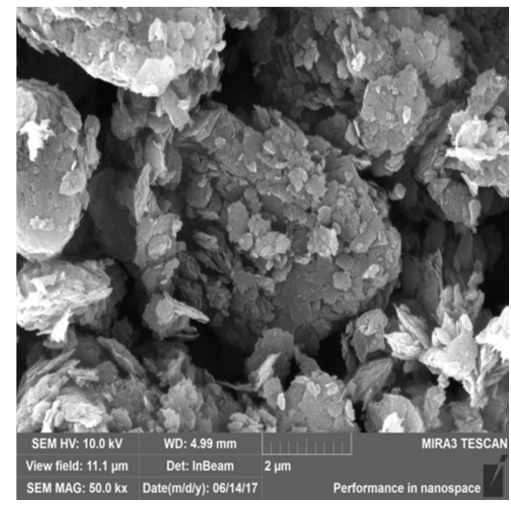

(b)

Figure 3. SEM images of graphene oxides: (a) GO I; (b) GO II and (c) GO-SA.

Above mentioned results and stated conclusions concerning the stability of undamaged oxidized plates are also in corespondence with the results of thermal analysis showing better thermal stability of GO I and GO II (Table 2). Beginning of the decomposition was observed at significantly higher temperatures than in case of GO-SA. Slightly lower temperature of the beginning of decomposition can be observed in case of GO I probably due to an idea of homogeneous distribution of oxygen in the structure as it was proposed when discussing results of elemental analysis.

Table 2. Thermal analysis results of graphene oxides.

\begin{tabular}{ccc}
\hline & \multicolumn{2}{c}{ Decomposition $\left({ }^{\circ} \mathrm{C}\right)$} \\
\cline { 2 - 3 } & Beginning & Inflexion \\
\hline GO I & 550 & 725 \\
\hline GO II & 630 & 745 \\
\hline GO-SA & 355 & 580 \\
\hline
\end{tabular}


Also investigations into the crystal structure of products (including GO-SA) were performed using X-ray diffraction analysis (XRD) but all of the results showed unaltered patterns of a graphitic structure with dominant peak at ca $2 \theta=26.4^{\circ}$. No significant changes of interplanar distances were observed and thus it can be stated that oxygen containing groups are bound especially on edges, eventually close to carbon layers, and they do not cause increase of distances among the planes.

Prepared and characterized graphene oxides GO I and GO II were subsequently chemically modified to reduced graphene oxides using literature methods [3,7] when hydrazine hydrate (further denoted as rGO X.I, where $X$ indicates original GO) and ascorbic acid (rGO X.II) were employed as the most suitable reducing agents.

Two pairs of reduced graphene oxides (rGO) were prepared from original graphene oxides using both of the reducing agents. As expected we observe decrease in oxygen content in case of all of the prepared rGOs althought it is not very significant (Table 3). However oxygen content in prepared rGOs is much lower than in case of standard rGO-SA (declared as hydrazine reduced) which shows significantly higher content of C-O bonds (even higher than in graphene oxide GO-SA). This is also reflected in the results of elemental analysis.

Table 3. Reduced graphene oxides - elemental analysis and XPS analysis results.

\begin{tabular}{cccccccc}
\hline & $\begin{array}{c}\text { Formula } \\
\text { (EA) }\end{array}$ & \multicolumn{5}{c}{ C1s, specific groups abundance (rel. \%) } \\
& & $\mathrm{C}=\mathrm{C}$ & $\mathrm{C}-\mathrm{C}$ & $\mathrm{C}-\mathrm{O}$ & $\mathrm{C}=\mathrm{O}$ & $\mathrm{C}(\mathrm{O}) \mathrm{O}$ & sum C O \\
\hline rGO I.I & $\mathrm{C}_{16} \mathrm{O}$ & 62.50 & 26.92 & 6.02 & 2.55 & 1.99 & $\mathbf{1 0 . 5 6}$ \\
\hline rGO II.I & $\mathrm{C}_{21} \mathrm{O}$ & 60.96 & 29.24 & 5.44 & 2.04 & 2.32 & $\mathbf{9 . 8 0}$ \\
\hline rGO I.II & $\mathrm{C}_{27} \mathrm{O}$ & 61.29 & 28.88 & 5.84 & 2.33 & 2.00 & $\mathbf{1 0 . 1 7}$ \\
\hline rGO II.II & $\mathrm{C}_{21} \mathrm{O}$ & 63.23 & 25.08 & 7.12 & 2.30 & 2.28 & $\mathbf{1 1 . 7 0}$ \\
\hline rGO-SA & $\mathrm{C}_{8} \mathrm{O}$ & 61.74 & 21.16 & 12.19 & 2.93 & 1.99 & $\mathbf{1 7 . 1 1}$ \\
\hline
\end{tabular}

Above mentioned differences are evident when comparing Raman spectra (Figure 4) as rGO-SA spectrum shows a significant D-peak $\left(\mathrm{ca} 1350 \mathrm{~cm}^{-1}\right.$ ) having even higher intensity than G-peak (ca $1580 \mathrm{~cm}^{-1}$ ) evincing very high level of carbon layers damage. Thus we can claim that rGOs prepared from GO I and GO II are not further disrupted during the reduction process and thus keeping carbon layers in a fine quality of the starting graphene oxide. We can also observe that reduction using ascorbic acid is generaly more gentle to carbon layers as D-peak has a very low intensity in case of rGO I.II and rGO II.II.

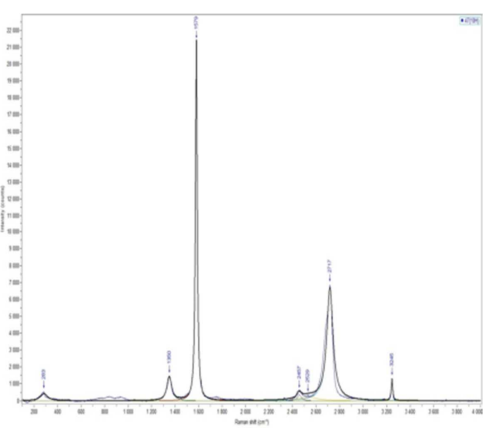

(a)

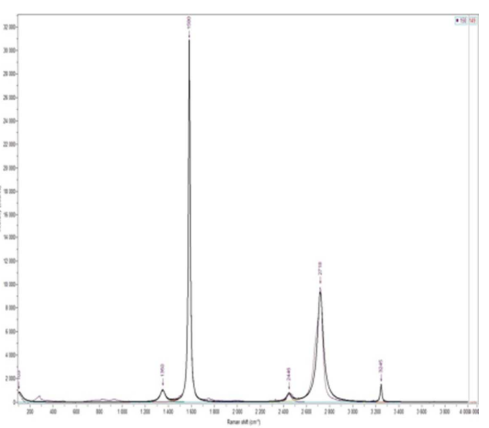

(b)

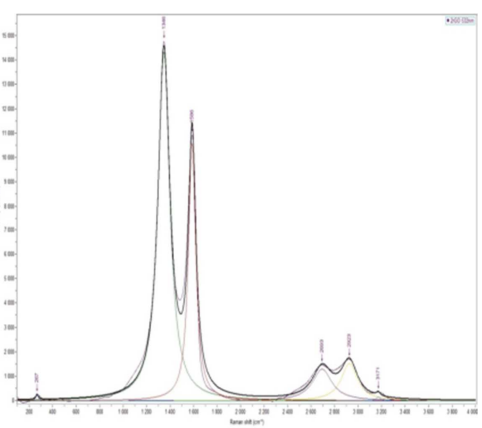

(c)

Figure 4. Raman spectra of reduced graphene oxides: (a) rGO I.I; (b) rGO I.II and (c) rGO-SA. 
FT-IR spectra (Figure 4), similarly as in case of graphene oxides, suffer from low intensities although we can see bands at ca $1600 \mathrm{~cm}^{-1}$ ( $\mathrm{C}=\mathrm{C}$ bonds) and ca $1700 \mathrm{~cm}^{-1}(\mathrm{C}=\mathrm{O}$ bonds). We can observe very low intensity of $\mathrm{C}=\mathrm{O}$ bands especially in case of ascorbic acid reduced products rGO I.II and rGO II.II. In case of rGO-SA bands at ca $1630 \mathrm{~cm}^{-1}$ lose their intensity while bands at ca $1560 \mathrm{~cm}^{-1} \mathrm{become}$ the most prominent.

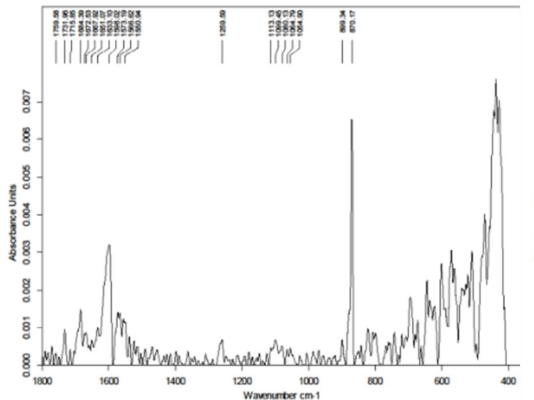

(a)

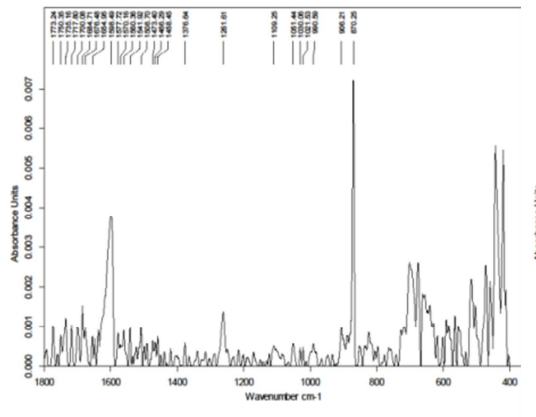

(b)

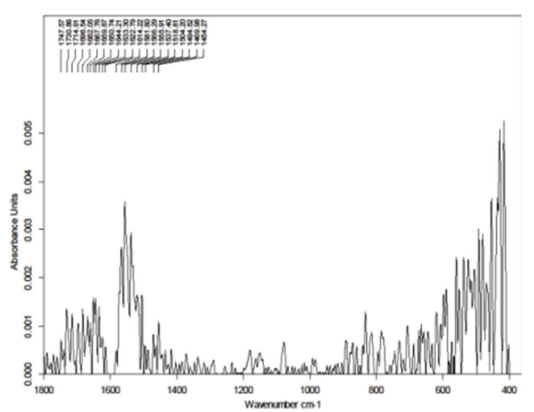

(c)

Figure 4. FT-IR spectra of reduced graphene oxides: (a) rGO I.I; (b) rGO I.II and (c) rGO-SA.

Preliminary results are also confirmed by scanning electron microscopy (Figure 5). Images of reduced graphene oxides prepared from GO I and GO II show large and undamaged plates while in case of rGO-SA we can observe significantly different appearance of the sample. Again we can mention slightly less fragmentation in case of ascorbic acid reduced products rGO I.II and rGO II.II.

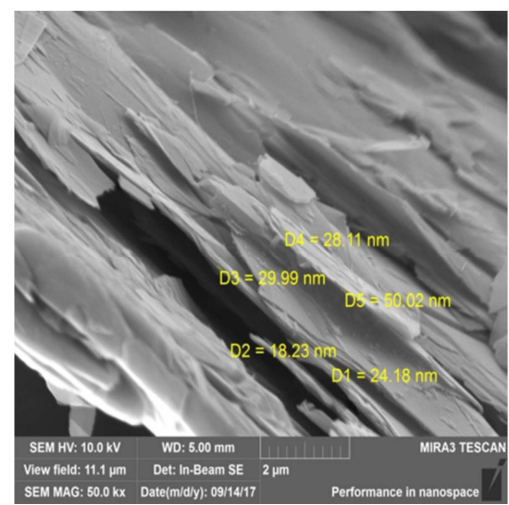

(a)

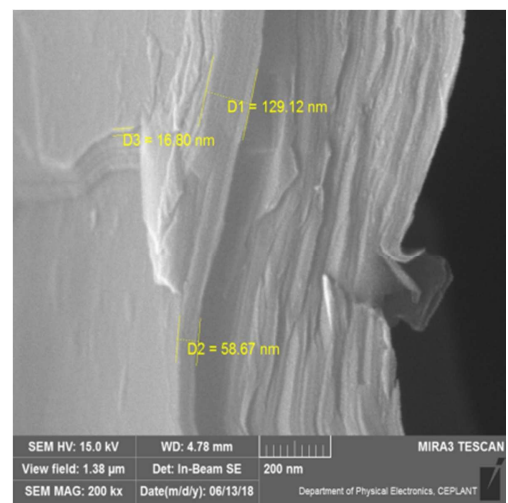

(b)

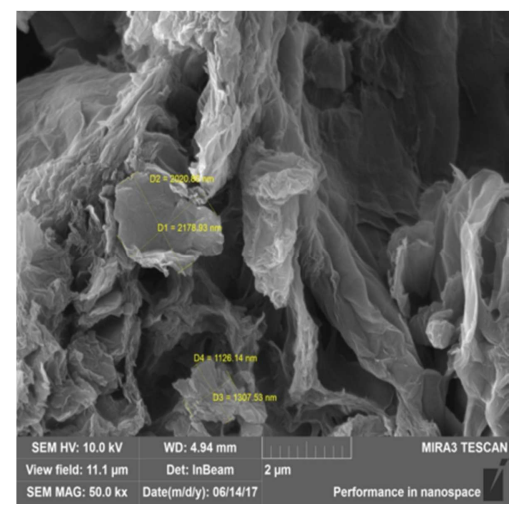

(b)

Figure 5. SEM images of reduced graphene oxides: (a) rGO I.I; (b) rGO I.II and (c) rGO-SA.

Thermal analysis of reduced graphene oxides brought similar results regarding comparison among prepared products and the standard (Table. 4) althought difference is not so significant as in the case of graphene oxides. Only slightly better thermal stability can be considered rather unexpected as on the basis of above mentioned results we expected more significant differences.

Table 4. Thermal analysis results of reduced graphene oxides.

\begin{tabular}{ccc}
\hline & \multicolumn{2}{c}{ Decomposition $\left({ }^{\circ} \mathrm{C}\right)$} \\
\cline { 2 - 3 } & Beginning & Inflexion \\
\hline rGO I.I & 560 & 740 \\
\hline rGO II.I & 580 & 680 \\
\hline rGO I.II & 600 & 770 \\
\hline rGO II.II & 550 & 730 \\
\hline rGO-SA & 510 & 600 \\
\hline
\end{tabular}


On the contrary results of X-ray diffraction analysis of new products and standard differ significantly (Figure 6). While prepared products still keep graphite diffraction patterns with prominent peak at $2 \theta=26.4^{\circ}$, standard rGO-SA shows additional new peaks at lower angles $(2 \theta=$ $22.4^{\circ}$ and $21.3^{\circ}$ ) indicating some increase in interplanar distances probably due to a higher level of oxidation or other structural changes caused by reduction/oxidation processes.

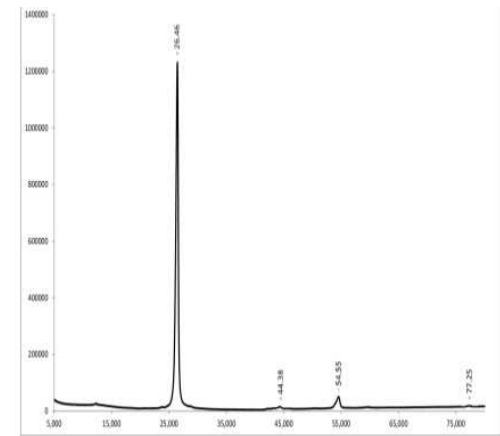

(a)

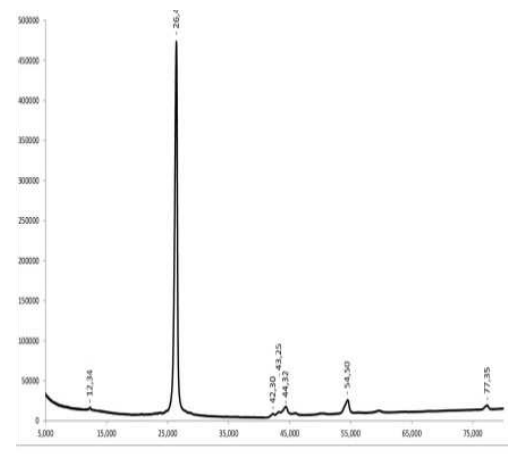

(b)

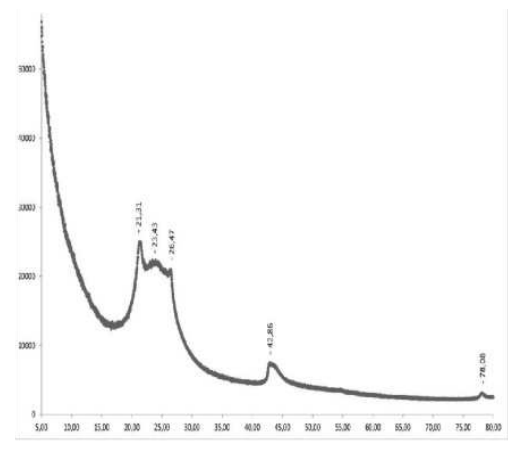

(b)

Figure 6. XRD diffractograms of reduced graphene oxides: (a) rGO I.I; (b) rGO I.II and (c) rGO-SA.

\section{Conclusion}

As a result of our recent study we present characterization data of a series of new graphene-like materials. Graphene oxides were prepared according to a new and cheap method following principles of green and sustainable chemisty which is a subject of a classified know-how of Masaryk University. Moreover a simple experimental scheme allows an easy expansion into industrial scale. Modification to reduced graphene oxides was achieved using literature methods and further research concerning other derivatives of graphene oxides, e.g. amides, esters etc., is still in progress.

On the basis of results of a thorough characterization using a variety of methods we can claim that generally new graphene-like products do not suffer from the utilization of harsh oxidizing agents and strongly acidic solutions and they feature large and unaltered layers of $\mathrm{sp}^{2}$ carbons which were not damaged during reactions. The fact can be considered as very important as unique features of graphene arise from structural and electronic (aromaticity) arrangement of the carbon layers. Additionally products are free of impurities and low molecular weight carbon fragments although they keep the same level of oxidation. Distribution of oxygen containg groups can be varied using different oxidizing agents. Variations on oxygen containing groups can be with advantage utilized in practical applications and in further research. Low damage of carbon layers is then reflected in higher thermal stability of the compounds as well.

All of the products can be utilized in a chemical research as well as in a variety of practical or industrial applications such as electrical engineering and electronics, energy storage, adsorption and catalysis, medicinal and biochemical applications and material science. At the time, described products can be purchased via Masaryk university on request, commercial marketing is now under intense negotiation.

\section{Patents}

New methods of graphene oxide preparation are a subject of a classified know-how of Masaryk University as an originator. 
Author Contributions: chemical experiments, research, D.N.; research, analytics, data analysis, writing-original draft preparation, R.Š.; conceptualization, review and editing, P.P.

Funding: This research was funded by the donation of a private company Senergos a.s., Czech Republic.

Acknowledgments: We would like to thank our colleagues at R\&D Centre for Low-Cost Plasma and Nanotechnology Surface Modifications, Faculty of Science, Masaryk University, for a kind help with analytical measurements.

Conflicts of Interest: The authors declare no conflict of interest.

\section{References}

1. Mohan, V.B.; Lau, K.; Hui, D.; Bhattacharyya, D. Graphene-based materials and their composites: A review on production, applications and product limitations. Composites Part B 142 2018, 200-220. DOI: 10.1016/j.compositesb.2018.01.013.

2. Dreyer, D.R.; Todd, A.D.; Bielawski Ch.W. Harnessing the chemistry of graphene oxide. Chem. Soc. Rev. 2014, 43, 5288-5301, DOI: 10.1039/c4cs00060a.

3. Li, J.; Zeng, X.; Ren, T.; Heide van der, E. The Preparation of Graphene Oxide and Its Derivatives and Their Application in Bio-Tribological Systems. Lubricants 2014, 2, 137-161; DOI: 10.3390/lubricants2030137.

4. Eigler, S. Controlled Chemistry Approach to the Oxo-Functionalization of Graphene. Chem. Eur. J. 2016, 22, 7012 - 7027, DOI: 10.1002/chem.201600174.

5. Georgakilas, V.; Tiwari, J.N.; Kemp, K.Ch.; Perman, J.A.; Bourlinos, A.B.; Kim, K.S.; Zboril, R. Noncovalent Functionalization of Graphene and Graphene Oxide for Energy Materials, Biosensing, Catalytic, and Biomedical Applications. Chem. Rev. 2016, 116, 5464-5519, DOI: 10.1021/acs.chemrev.5b00620.

6. Dimov, D.; Amit, I.; Gorrie, O.; Barnes, M.D.; Townsend, N.J.; Neves, A.I.S.; Withers, F.; Russo, S.; Craciun, M.F. Ultrahigh Performance Nanoengineered Graphene-Concrete Composites for Multifunctional Applications. Adv. Funct. Mater. 2018, 28, 1705183, DOI: 10.1002/adfm.201705183.

7. De Silva, K.K.H.; Huang, H.-H.; Joshi, R.K., Yoshimura, M. Chemical reduction of graphene oxide using green reductants. Carbon 2017, 119, 190-199, DOI: 10.1016/j.carbon.2017.04.025.

8. Bourlinos, A.B.; Gournis, D.; Petridis, D.; Szabó, T.; Szeri, A.; Dékány, I. Graphite Oxide: Chemical Reduction to Graphite and Surface Modification with Primary Aliphatic Amines and Amino Acids. Langmuir 2003, 19, 6050-6055, DOI: 10.1021/la026525h.

9. del Prado Lavin-Lopez, M.; Romero, A.; Garrido, J.; Sanchez-Silva, L.; Valverde, J. L. Influence of Different Improved Hummers Method Modifications on the Characteristics of Graphite Oxide in Order to Make a More Easily Scalable Method. Ind. Eng. Chem. Res. 2016, 55, 12836-12847, DOI: 10.1021/acs.iecr.6b03533.

10. Muzyka, R.; Kwoka, M.; Smędowski , L.; Díez, N.; Gryglewicz, G. Oxidation of graphite by different modified Hummers methods. New Carbon Materials 2017, 32(1), 15-20, DOI: 10.1016/S1872-5805(17)60102-1.

11. Ferrari, A.C.; Meyer, J.C.; Scardaci, V.; Casiraghi, C.; Lazzeri, M.; Mauri, F.; Piscanec, S.; Jiang, D.; Novoselov, K.S.; Roth, S.; Geim, A.K. Raman Spectrum of Graphene and Graphene Layers. Phys. Rev. Lett. 2006, 97, 187401, DOI: 10.1103/PhysRevLett.97.187401.

12. Akhavan, O.; Abdolahad, M.; Esfandiar, A.; Mohatashamifar, M. Photodegradation of Graphene Oxide Sheets by $\mathrm{TiO}_{2}$ Nanoparticles after a Photocatalytic Reduction. J. Phys. Chem. C 2010, 114, 12955-12959, DOI: $10.1021 /$ jp103472c.

(c) 2019 by the authors. Submitted for possible open access publication under the terms and conditions of the Creative Commons Attribution (CC BY) license (http://creativecommons.org/licenses/by/4.0/). 